\title{
Up to $40 \%$ of COVID-19 Critically Ill Patients Have Vitamin D Deficiency
}

\author{
Teresa-Maria Tomasa-Irriguible ${ }^{1, *}$, Lara Bielsa-Berrocal ${ }^{2}$ and Luisa Bordejé Laguna ${ }^{3}$ \\ 1 Germans Trias i Pujol Hospital \\ 2 Germans Trias i Pujol Hospital; larabielsa@gmail.com \\ 3 Germans Trias i Pujol Hospital; luisabordeje@gmail.com \\ * Correspondence: teresatomasa@gmail.com
}

\begin{abstract}
Background: Coronavirus disease (COVID-19) has caused more than 745,000 deaths worldwide. Vitamin D has been identified as a potential strategy to prevent or treat this disease. The purpose of the study was to measure vitamin D at hospital admission of COVID-19; Methods: We included critically ill patients with the polymerase chain reaction positive test for COVID-19, from March to April, 2020. Statistical significance was defined as $\mathrm{P}<.05$. All tests were 2-tailed; Results: A total of 35 patients (median age, 60 years; 26 [74.3\%] male) were included. Vitamin D levels were categorized as deficient for 14 participants $(40 \%)$. Vitamin D deficiency was associated with vitamin $\mathrm{A}(\mathrm{P}=0.003)$ and Zinc $(\mathrm{P}=0.019)$ deficiency and lower levels of albumin $(\mathrm{P}=0.026)$ and prealbumin $(\mathrm{P}=0.009)$. Overall, none of the studied variables were associated with vitamin $\mathrm{D}$ status: mortality, intensive care unit (ICU) or hospital stay, necessity of vasoactive agents, intubation, prone position, $C$ reactive protein (CRP), Dimer-D, Interleukin 6 levels (IL-6), ferritin levels, or bacterial superinfection; Conclusions: In this single-center, retrospective cohort study, deficient vitamin D status was found in 40\% in COVID-19 critically ill patients. However, deficient vitamin $\mathrm{D}$ status was not associated with inflammation or outcome.
\end{abstract}

Keywords: SARS-CoV-2; covid-19; vitamins

\section{Introduction}

Coronavirus disease 2019 (COVID-19) has caused more than 745000 deaths worldwide [1]. Patients with COVID-19 show clinical clusters of severe respiratory illness manifestations including fever, nonproductive cough, dyspnea, myalgia, fatigue, abnormal leukocyte counts, and radiographic evidence of pneumonia, which are similar to the symptoms of previous SARS-CoV and MERS-CoV infections [2]. SARS-CoV-2 infection can remain asymptomatic or cause modest symptoms. Severely sick patients require hospital admission and about $20 \%$ of hospitalized patients will developed Acute Respiratory Distress Syndrome (ARDS) and require intensive care unit (ICU) treatment [3]. ARDS, also in patients with Coronavirus Disease 2019 (COVID-19) is a life-threatening condition [4,5]. Although frequencies vary according to series, more than $40 \%$ of patients hospitalized because of COVID-19 pneumonia developed ARDS of which more than $50 \%$ ultimately died [6]. Vitamin D treatment has been identified as a potential strategy to prevent or treat COVID-19 [7]. There is not enough evidence on the association between vitamin D levels and COVID-19 severity and mortality [8]. Therefore, studies are necessary to test this hypothesis.

\section{Materials and Methods}

This is a retrospective cohort study at a single ICU. Vitamin D deficiency was defined by a measurement of 25-hydroxycholecalciferol below than $10 \mathrm{ng} / \mathrm{mL}$ in the first 24- hour after COVID-19 testing. We included critically ill adult patients with COVID-19 who met ARDS criteria according to the Berlin definition [9] at ICU from March to April, 2020. We obtained electronic health record data for demographic, comorbidity and laboratory. This study was approved by the Investigation Ethics 
Committee of the Hospital (PI-20-253) with a waiver of consent for use of identifiable data. This study followed the Strengthening the Reporting of Observational Studies in Epidemiology (STROBE) reporting guideline for cohort studies. All variables were defined based on information from the UCM electronic health record (Epic; Epic Systems). COVID-19 test status was determined by any positive COVID-19 polymerase chain reaction test result. Basic descriptive statistics were reviewed for all variables. Fisher exact test was used for binary variables and the $t$ test for continuous variables. Statistical significance was defined as $\mathrm{P}<.05$. All tests were 2 -tailed.

\section{Results}

A total of 35 patients (median age, 60 years; 26 [74.3\%] male) had a vitamin D status at first 24-hour after COVID-19 testing was categorized as deficient for 14 participants $(40 \%)$ and insufficient for 25 (71.4\%). Vitamin D deficiency was associated with vitamin A ( $P=0.003)$ and Zinc $(P=0.019)$ deficiency and lower levels of albumin $(P=0.026)$ and prealbumin $(P=0.009)$. Overall, none of the studied variables were associated with vitamin D status: Vitamins B, C and E, mortality, intensive care unit (ICU) or hospital stay, necessity of vasoactive agents, intubation, prone position, renal replacement therapies (RRT), extracorporeal membrane oxygenation (ECMO), nor reactive $C$ protein (RCP), Dimer-D, Interleukin 6 levels (IL-6), ferritin levels, nor complications as acute kidney injury (AKI), or bacterial superinfection. See table1.

Table 1. Characteristics of Patient Population.

\begin{tabular}{cc}
\hline Clinical characteristics & COVID-19 ARDS (n=35) \\
\hline Age (median, min-max, years) & $60(33-76)$ \\
Male (n, \%) & $26(74.3)$ \\
SOFA score (median, min-max, points) & $7(2-15)$ \\
Intubation (n, \%) & $29(82.6)$ \\
Prone position (n, \%) & $27(77.14)$ \\
VV-ECMO (n, \%) & $3(8.57)$ \\
VA-ECMO (n, \%) & $1(2.86)$ \\
Noradrenaline (n, \%) & $25(71.4)$ \\
Dobutamine (n, \%) & $2(5.7)$ \\
CKD (n, \%) & $3(8.57)$ \\
AKI (n, \%) & $15(42.86)$ \\
CRRT (n, \%) & $1(2.86)$ \\
IRR (n, \%) & $1(2.86)$ \\
DVT (n, \%) & $13(37.14)$ \\
PE (n, \%) & $411.4)$ \\
Bacterial superinfection (n, \%) & $1(2.86)$ \\
ICU-LOS (median, min-max, days) & $12(1-112)$ \\
HOSP-LOS (median, min-max, days) & $25(1-185)$ \\
Mortality (n, \%) & $15(27.8)$ \\
IL-6 (median, min-max, pg/mL, Normal values: 0 - 6.4) & $12.8(4-34.1)$ \\
Ferritin (median, min-max, ng/mL, Normal values: $30-400)$ & $25(71.4)$ \\
Dimer-D (median, min-max, ng/mL, Normal values: 0 - 500) & $14(40)$ \\
\hline
\end{tabular}

SOFA: sequential organ failure assessment; ICU: intensive care unit; ECMO: extracorporeal membrane oxygenation; CKD: chronic kidney disease; AKI: acute kidney injury; CRRT: continuous 
renal replacement therapy; IRR intermittent renal replacement; DVT: deep venous thrombosis; PE: pulmonary embolism; ICU-LOS: Length of ICU stay; HOSP-LOS: Length of hospital stay; Vit. D: Vitamin D levels; IL-6: Interleukin 6 levels; RCP: reactive C protein.

\section{Discussion}

In this single-center retrospective cohort study, deficient vitamin D status was found in up to $40 \%$ in COVID-19 critically ill patients with ARDS. Patients with severe ARDS [10,11] or requiring ICU [12] are frequently severely vitamin D deficient [13]. In addition, low serum 25-hydroxyvitamin $\mathrm{D}(25[\mathrm{OH}] \mathrm{D})$ levels in patients hospitalized with COVID-19 are associated with greater disease severity [14]. Vitamin D treatment has been found to decrease other viral respiratory infections, especially in persons with vitamin D deficiency [15]. Vitamin D modulates immune function through effects on dendritic cells and T cells [16], which may promote viral clearance and reduce inflammatory responses that produce symptoms. Given the low risks and low cost of vitamin D treatment, recent reporting has suggested that vitamin D treatment should be scaled [17]. Nevertheless, evidence of whether vitamin D deficiency is associated with COVID-19 infection and whether vitamin D treatment may help COVID-19 is lacking. In our study, vitamin D deficiency was a frequent event, but it was not associated with inflammation or outcome. Conversely, a pilot study with calcifediol has recently concluded that calcifediol may improve the clinical outcome of patients requiring hospitalization for COVID-19 [18].

Our study has also point out that Vitamin D deficiency was associated with other micronutrients deficiency as retinol and Zinc. Thus, Vitamin D deficiency is not an isolated event in COVID-19 with ARDS and is accompanied by other micronutrient deficiencies that should also be taken into account.

\section{Conclusions}

In this single-center retrospective cohort study, deficient vitamin D status was found in $40 \%$ in up to COVID-19 critically ill patients with ARDS. Vitamin D deficiency was associated with other micronutrients deficiency as retinol and Zinc. However, deficient vitamin D status was no associated with inflammation or outcome. Nonetheless, this was a small cohort study and vitamin D was measured in northern Spain in spring.

Author Contributions: Conceptualization, T.M.T.I.; methodology, L.B.B.; software, L.B.B.; validation, T.M.T.I.,; formal analysis, L.B.B.; investigation, T.M.T.I.; data curation, L.B.B.; writing-original draft preparation, L.B.B.; writing-review and editing, T.M.T.I.; supervision, T.M.T.I. All authors have read and agreed to the published version of the manuscript.

Funding: This research received no external funding.

Acknowledgments: We acknowledge the dedication, commitment, and sacrifice of the staff, and personnel in our institution through the Covid-19 crisis.

Conflicts of Interest: The authors declare no conflict of interest.

\section{References}

1. COVID-19 corona virus pandemic. Worldometer. Updated August 12, 2020. Accessed August 12, 2020. https:// www.worldometers.info/coronavirus.

2. C. Huang, Y. Wang, X. Li, L. Ren, J. Zhao, Y. Hu, L. Zhang, G. Fan, J. Xu, X. Gu, Z. Cheng, T. Yu, J. Xia, Y. Wei, W. Wu, X. Xie, W. Yin, H. Li, M. Liu, Y. Xiao, H. Gao, L. Guo, J. Xie, G. Wang, R. Jiang, Z. Gao, Q. Jin, J. Wang, B. Cao, Clinical features of patients infected with 2019 novel coronavirus in Wuhan, China, Lancet 395 (2020) 497-506, https://doi.org/10.1016/S0140-6736(20)30183-5.

3. N. Chen, M. Zhou, X. Dong, J. Qu, F. Gong, Y. Han, Y. Qiu, J. Wang, Y. Liu, Y. Wei, J. Xia, T. Yu, X. Zhang, L. Zhang, Epidemiological and clinical characteristics of 99 cases of 2019 novel coronavirus pneumonia in Wuhan, China: a descriptive study, Lancet 395 (2020) 507-513, https://doi.org/10.1016/S0140-6736(20)30211-7.

4. E. Fan, D. Brodie, A.S. Slutsky, Acute respiratory distress syndrome, JAMA 319 (2018) 698, https://doi.org/10.1001/jama.2017.21907 
5. V.M. Ranieri, G.D. Rubenfeld, B.T. Thompson, N.D. Ferguson, E. Caldwell, E. Fan, L. Camporota, A.S. Slutsky, Acute respiratory distress syndrome: the Berlin definition, JAMA - J. Am. Med. Assoc. 307 (2012) 2526-2533, https://doi.org/ 10.1001/jama.2012.5669.

6. C. Wu, X. Chen, Y. Cai, J. Xia, X. Zhou, S. Xu, H. Huang, L. Zhang, X. Zhou, C. Du, Y. Zhang, J. Song, S. Wang, Y. Chao, Z. Yang, J. Xu, X. Zhou, D. Chen, W. Xiong, L. Xu, F. Zhou, J. Jiang, C. Bai, J. Zheng, Y. Song, Risk factors associated with acute respiratory distress syndrome and death in patients with coronavirus disease 2019 Pneumonia in Wuhan, China, JAMA Intern. Med. (2020), https://doi.org/10.1001/ jamainternmed.2020.0994.

7. Grant WB, Lahore H, McDonnell SL, et al. Evidence that vitamin D supplementation could reduce risk of influenza and COVID-19 infections and deaths. Nutrients. 2020;12(4):988. doi:10.3390/nu12040988.

8. Ali N. Role of vitamin D in preventing of COVID-19 infection, progression and severity. J Infect Public Health (2020), https://doi.org/10.1016/j.jiph.2020.06.021.

9. ARDS Definition Task Force, Ranieri VM, Rubenfeld GD, Thompson BT, Ferguson ND, Caldwell E, Fan E, Camporota L, Slutksy AS. Acute respiratory distress syndrome: the Berlin definition. JAMA. 2012;307(23):2526-33.

10. W. Viechtbauer, L. Smits, D. Kotz, L. Bud'e, M. Spigt, J. Serroyen, R. Crutzen, A simple formula for the calculation of sample size in pilot studies, J. Clin. Epidemiol. 68 (2015) 1375-1379, https://doi.org/10.1016/j.jclinepi.2015.04.014.

11. S. Russo, L. Carlucci, C. Cipriani, A. Ragno, S. Piemonte, R. Del Fiacco, J. Pepe, V. Fassino, S. Arima, E. Romagnoli, S. Minisola, Metabolic changes following $500 \mu \mathrm{g}$ monthly administration of calcidiol: a study in normal females, Calcif. Tissue Int. 89 (2011) 252-257, https://doi.org/10.1007/s00223-011-9513-1.

12. Laboratory testing for 2019 novel coronavirus (2019-nCoV) in suspected human cases, (n.d.). https://www.who.int/publications/i/item/10665-331501 (accessed June 23, 2020).

13. E. Laird, J. Rhodes, R.A. Kenny, Vitamin D. And inflammation: potential implications for severity of Covid-19, Ir. Med. J. 113 (2020) (Accessed August 13, 2020), https://pubmed.ncbi.nlm.nih.gov/32603576/.

14. G. Panagiotou, S.A. Tee, Y. Ihsan, W. Athar, G. Marchitelli, D. Kelly, C.S. Boot, N. Stock, J. Macfarlane, A.R. Martineau, G. Burns, R. Quinton, Low serum 25- hydroxyvitamin D (25[OH]D) levels in patients hospitalised with COVID-19 are associated with greater disease severity, Clin. Endocrinol. (Oxf). (2020), https:// doi.org/10.1111/cen.14276.

15. Martineau AR, Jolliffe DA, Hooper RL, et al. Vitamin D supplementation to prevent acute respiratory tract infections: systematic review and meta-analysis of individual participant data. BMJ. 2017;356:i6583. doi:10.1136/ bmj.i6583.

16. Yin K, Agrawal DK. Vitamin D and inflammatory diseases.J Inflamm Res. 2014;7:69-87. doi:10.2147/JIR. S63898.

17. Medical societies advise on vitamin D in midst of COVID-19. Medscape. Published July 10, 2020. Accessed July 10, 2020. https://www.medscape.com/viewarticle/933715.

18. Entrenas Castillo M, Entrenas Costa LM, Vaquero Barrios JM, Alcala Díaz JF, Lopez Miranda J, Bouillon R, et al. Effect of calcifediol treatment and best available therapy versus best available therapy on intensive care unit admission and mortality among patients hospitalized for COVID-19: A pilot randomized clinical study. Journal of Steroid Biochemistry and Molecular Biology 203 (2020) 105751. 